

\title{
American and European Foulbrood In Colonies of Honey Bees In Connecticut
}

\author{
John F. Anderson and Harry K. Kaya
}

The Office of the State Entomologist, in aceordance with Section 22-89 of the General Statutes, inspects colonies of the honey bee, Apis mellifera L., for diseases, the most important of which are the bacterial diseases commonly known as foulbroods. Inspections have been carried out yearly since 1910 . In this Bulletin the incidence of loulbrood in 1974 is compared with the incidence in previous years.

\section{METHODS}

Bacillus larvae, which causes American foulbrood, kills mature larvae and young pupae after the brood cells have been sealed. Streptococcus pluton which causes European foulbrood usually kills young larvae.

In 1974, $6 \%$ of the registered colonies, selected at random in each county, were examined. Infections were identified by characteristic odors and were verified microscopically. Samples of brood comb were taken to the laboratory for examination. Dead larvae were examined for spores of $B$. larvae.

During the period 1910-1973 as many colonies as possible were examined by the inspectors. All known colonies were inspected from 1941-1946 and possibly in 1949. In the other years between 1910 and 1973 the inspectors tended to inspect colonies in areas where loulbrood was known to occur. Thus the disease incidence recorded for these years is higher than the actual level throughout the state.

\section{RESULTS}

In 1974, less than 1\% of the colonies examined were lound infected with American foulbrood (Table 1). This incidence was similar to rates of infection

Table 1. Incidence of Americon Foulbrood in Connecticut, 1974

\begin{tabular}{lcc} 
& \multicolumn{2}{c}{ No. of colonies } \\
\cline { 2 - 3 } County & Examined & Infected \\
\hline Litchfield & 68 & 0 \\
Hortford & 78 & 0 \\
Windhom & 28 & 0 \\
Tolland & 27 & 2 \\
Foirfield & 66 & 0 \\
New Hoven & 64 & 0 \\
Middlesex & 26 & 0 \\
New London & 66 & 2 \\
Totol & 423 & 4 \\
\hline
\end{tabular}

recorded in previous years (Table 2). Infected colonies were encountered in the 1974 random sample of hives only in Tolland and New London counties although American loulbrood occurs in all counties in Connecticut.

European loulbrood was prevalent at the time the inspection service was initiated in 1910 (Table 2). However, the disease declined rapidly until $1 \%$ or less of the inspected colonies were inlected in 1921 
Table 2. Incidence of American and Eurapean Foulbrood in Connecticut, 1910.1973*

\begin{tabular}{|c|c|c|c|c|c|c|c|c|c|c|c|c|c|}
\hline \multirow[b]{3}{*}{ Year } & \multicolumn{3}{|c|}{ Apiaries } & \multicolumn{3}{|c|}{ Colonies } & \multicolumn{4}{|c|}{ Apiaries } & \multicolumn{2}{|c|}{ Colonies } & \\
\hline & \multirow{2}{*}{$\begin{array}{c}\text { No. } \\
\text { inspected }\end{array}$} & \multicolumn{2}{|c|}{$\%$ infected } & \multirow{2}{*}{$\begin{array}{c}\text { No. } \\
\text { inspected }\end{array}$} & \multicolumn{2}{|c|}{$\%$ infected } & \multirow[b]{2}{*}{ Year } & \multirow{2}{*}{$\begin{array}{c}\text { No. } \\
\text { inspected }\end{array}$} & \multicolumn{2}{|c|}{$\%$ infected } & \multirow{2}{*}{$\begin{array}{l}\text { Na. } \\
\text { inspected }\end{array}$} & \multirow{2}{*}{\multicolumn{2}{|c|}{$\frac{\% \text { infected }}{\text { EFB AFB }}$}} \\
\hline & & EFB & $\mathrm{AFB}$ & & EFB & $\overline{\mathrm{AFB}}$ & & & EFB & AFB & & & \\
\hline 1910 & 208 & 76 & 0 & 1595 & 50 & 0 & 1943 & 2635 & 0 & 7 & 14903 & 0 & 3 \\
\hline 1911 & 162 & 52 & 0 & 1571 & 27 & 0 & 1944 & 2451 & $<0.1$ & 8 & 12360 & $<0.1$ & 3 \\
\hline 1912 & 153 & 48 & 0 & 1431 & 24 & 0 & 1945 & 2589 & $<0.1$ & 6 & 13353 & $<0.1$ & 2 \\
\hline 1913 & 189 & 44 & 0 & 1500 & 25 & 0 & 1946 & 2827 & 0 & 6 & 11920 & 0 & 2 \\
\hline 1914 & 463 & 33 & 1 & 3882 & 14 & 1 & 1947 & 1925 & 0 & 6 & 9648 & 0 & 3 \\
\hline 1915 & 494 & 26 & 1 & 4241 & 10 & 0.2 & 1948 & 2165 & $<0.1$ & 7 & 11837 & 0.1 & 2 \\
\hline 1916 & 467 & 19 & I & 3898 & 7 & 0.2 & 1949 & 2423 & 0 & 8 & 13416 & 0 & 3 \\
\hline 1917 & 473 & 17 & 0.4 & 4506 & 5 & 0.2 & 1950 & 2277 & $<0.1$ & 7 & 12899 & $<0.1$ & 2 \\
\hline 1918 & 395 & 10 & 1 & 3047 & 3 & 0.3 & 1951 & 2632 & 0 & 4 & 13895 & 0 & 1 \\
\hline 1919 & 723 & 7 & 3 & 6070 & 1 & 1 & 1952 & 2299 & 0.1 & 6 & 9691 & $<0.1$ & 3 \\
\hline 1920 & 762 & 4 & 1 & 4797 & 2 & 0.3 & 1953 & 2324 & 0 & 6 & 11406 & 0 & 3 \\
\hline 1921 & 751 & 4 & 2.5 & 6972 & 1 & 1 & 1954 & 2201 & 0 & 5 & 11605 & 0 & 2 \\
\hline 1922 & 797 & 4 & 1 & 8007 & 1 & 0.3 & 1955 & - & - & - & 13166 & - & 1 \\
\hline 1923 & 725 & 2 & 1 & 6802 & 0.4 & 0.3 & 1956 & 2316 & 0.1 & 3 & 9294 & $<0.1$ & 1 \\
\hline 1924 & 953 & 2 & 1 & 8929 & 1 & 0.2 & 1957 & 2355 & $<0.1$ & 3 & 9253 & $<0.1$ & 1 \\
\hline 1925 & 766 & 2 & 3 & 8257 & 1 & 0.5 & 1958 & 2218 & 0.1 & 4 & 9712 & 0.1 & 1 \\
\hline 1926 & 814 & 3 & 2 & 7923 & 1 & 0.3 & 1959 & 2409 & 0 & 3 & 10146 & 0 & 1 \\
\hline 1927 & 803 & 1 & 3 & 8133 & 0.3 & 1 & 1960 & 2425 & $<0.1$ & 3 & 9517 & $<0.1$ & 1 \\
\hline 1928 & 852 & 1 & 4 & 8023 & 0.3 & 1 & 1961 & 1720 & 0 & 1 & 7013 & 0 & 0.5 \\
\hline 1929 & 990 & 0.2 & 5 & 9559 & $<0.1$ & 1 & 1962 & 1702 & 0 & 3 & 7186 & 0 & 1 \\
\hline 1930 & 1059 & 0.3 & 5 & 10335 & $<0.1$ & 1 & 1963 & 1737 & 0 & 3 & 7371 & 0 & 1 \\
\hline 1931 & 1232 & 0.1 & 3 & 10678 & $<0 . i$ & 1 & 1964 & 1583 & 0 & 3 & 7198 & 0 & 1 \\
\hline 1932 & 1397 & 0 & 4 & 11459 & 0 & 1 & 1965 & 1700 & 0 & 3 & 7572 & 0 & 1 \\
\hline 1933 & 1342 & 0 & 2 & 10927 & 0 & 0.5 & 1966 & 1690 & 0 & 3 & 7179 & 0 & 1 \\
\hline 1934 & 1429 & 0.1 & 2 & 7128 & $<0.1$ & 1 & 1967 & 2073 & $<0.1$ & 3 & 7800 & $<0.1$ & 1 \\
\hline 1935 & 1333 & 0 & 6 & 8855 & 0 & 2 & 1968 & 1655 & 0 & 4 & 6668 & 0 & 2 \\
\hline 1936 & 1438 & 0.1 & 6 & 9278 & $<0.1$ & 2 & 1969 & 1520 & 0 & 4 & 7241 & 0 & 2 \\
\hline 1937 & 1438 & 0 & $\begin{array}{l}7 \\
0\end{array}$ & 10253 & 0 & 2 & 1970 & 1396 & 0 & 3 & 4983 & 0 & 2 \\
\hline 1938 & 1609 & 0 & $\begin{array}{l}8 \\
5\end{array}$ & 10705 & 0 & 2 & 1971 & 1592 & 0 & 3 & 6157 & 0 & 1 \\
\hline 1939 & 1627 & 0 & 3 & 8936 & 0 & 2 & 1972 & 1301 & 0 & 3 & 5184 & 0 & 1 \\
\hline 1940 & 1719 & 0 & 9 & 8552 & 0 & 4 & 1973 & 651 & 0.2 & 7 & 3013 & $<0.1$ & 3 \\
\hline $\begin{array}{l}1941 \\
1942\end{array}$ & $\begin{array}{l}2222 \\
2354\end{array}$ & $\begin{array}{l}0 \\
0\end{array}$ & $\begin{array}{l}8 \\
10\end{array}$ & $\begin{array}{l}10720 \\
13777\end{array}$ & $\begin{array}{l}0 \\
0\end{array}$ & $\begin{array}{l}4 \\
4\end{array}$ & "Doto & ren from & the or & ual & $t$ of the $\mathrm{O}$ & ffice of & the Stote \\
\hline
\end{tabular}

and thereafter. European foulbrood was not found in the random sample of colonies.

American foulbrood was first found in Connecticut in 1914 (Table 2). Incidence of colony infection rose to $4 \%$ in the $1940 \mathrm{~s}$, but has fluctuated from less than $1 \%$ to $3 \%$ since then. The percentage of American foulbrood-infected apiaries reached as high as $10 \%$ in 1942, although in more recent years the incidence has averaged $4 \%$.

Table 3 shows the average annual incidence of foulbrood in Connecticut by county from 1910-73. Incidence was highest in Fairfield County and lowest in Windham and Tolland Counties.

It is apparent that the incidence of American foulbrood has not increased in the past 30 or more years, although the disease is present in all parts of the state and is a potential hazard to beekeepers. European loulbrood is now rarely encountered in Connecticut.
Table 3. Averoge Annual Incidence of American faulbraad in Connecticut Calanies of Honey Bees, 1914-1973, by County.

\begin{tabular}{lcc}
\hline & \multicolumn{2}{c}{ Percent infected } \\
County & Apiories & Colanies \\
\hline Litchfield & 3.9 & 1.4 \\
Hortford & 3.4 & 1.2 \\
Windham & 2.3 & 0.8 \\
Tolland & 2.1 & 1.0 \\
Foirlield & 7.3 & 2.3 \\
New Hoven & 6.4 & 2.0 \\
Middlesex & 3.7 & 1.3 \\
New Landon & 6.3 & 1.8 \\
\hline
\end{tabular}

\section{ACKNOWLEDGMENTS}

Emil Pagan inspected the bee hives in the field, Mrs. Bonnie Hamid summarized the rates of foulbrood infection from the annual reports of the State Entomologist, and George Schuessler faithfully maintained the annual inspection records. We thank Dr. David Newton, Central Connecticut State College, for reviewing the manuscript. 


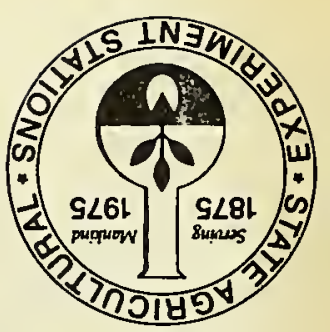

11ซ8 xาก

iVIว व४וн1

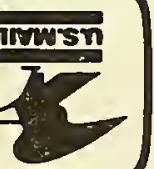

101 ชดซ

มมกเากวเมตร

Jo INJWLYYd 30 'S.

aIVd S33A aNY 3OVISOd

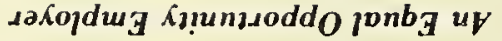

\author{
SL6L '3NกR \\ ZSL NiL317ng

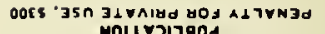 \\ Moltriาgend$$
\text { mobhom? ? }
$$ \\ ROS90 INJILJANNOJ NJAVH MJN

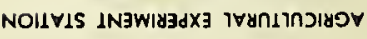 \\ InDIIDANNOS 3 HI
}


Sty $y_{5}$ Connecticut Libraries 
(1)

(n) 